\title{
The Influence of Methyl Jasmonate and Salicylic Acid on Secondary Metabolite Production in Rehmannia Glutinosa Libosch. Hairy Root Culture
}

\author{
Ewelina PiąTczaK*, Łukasz KuŹma, Halina Wysokińska \\ Department of Biology and Pharmaceutical Botany, Medical University of Łódź, \\ Muszyńskiego 1, 90-151 Łódź, Poland
}

Received November 4, 2015; revision accepted February 1, 2016

\begin{abstract}
Rehmannia glutinosa hairy roots were used to evaluate the effect of methyl jasmonate (MeJa) and salicylic acid (SA) on increase of root biomass and production of iridoids (catalpol, harpagide) and phenylethanoids (verbascoside and isoverbascoside). The elicitors were added to 23-day-old culture separately at concentrations between 50 and $200 \mu \mathrm{M}$ or in combinations at concentrations of 50 and $100 \mu \mathrm{M}$. Roots were harvested $72 \mathrm{~h}$ and $120 \mathrm{~h}$ after elicitation. The type of elicitor, its concentration and exposure time were found to strongly affect the content of each analyzed compound. A 72-hour treatment with $200 \mu \mathrm{M}$ MeJa was the most effective in increase of verbascoside content $\left(60.07 \mathrm{mg} \cdot \mathrm{DW}^{-1}\right.$ equivalent to $\left.845.45 \mathrm{mg} \cdot \mathrm{L}^{-1}\right)$ and isoverbascoside $\left(1.77 \mathrm{mg} \cdot \mathrm{DW}{ }^{-1}\right.$ equivalent to $24.94 \mathrm{mg} \cdot \mathrm{L}^{-1}$ ): these respective amounts were roughly 10- and 6.4-fold higher than the control values (unelicited roots). Exposure to $150 \mu \mathrm{M}$ MeJa provided optimal harpagide content after 72 hours $\left(0.136 \mathrm{mg}^{-\mathrm{DW}^{-1} ; 7.5 \text {-fold }}\right.$ increase compared to the control), and catalpol content after 120 hours (up to $2.145 \mathrm{mg} \cdot \mathrm{DW}^{-1}$ ). The combination of MeJa and SA also resulted in higher levels of secondary metabolites compared to the control culture, although these levels were lower than those observed for MeJa alone at the optimal concentration and exposure time. SA alone was less efficient in enhancing metabolite production than MeJa.
\end{abstract}

Keywords: elicitation, catalpol, hairy roots, harpagide, verbascoside, isoverbascoside

\section{INTRODUCTION}

Rehmannia glutinosa Libosch., a member of the Orobanchaceae family (Angiosperm Phylogeny Group III, 2009), is a perennial plant, which naturally occurs in China, Japan and Korea. It is one of the 50 fundamental herbs used in traditional Chinese medicine. Depending on the various processing methods described in the Chinese Pharmacopoeia (2000), Rehmanniae radix (Di Huang) is classified into three types: fresh root, dried root and steamed root. These types of roots are widely used as tonic, antianemic, antipyretic, antisenescence, antiulcer and antitumor agents (Kitagawa et al., 1991; Zhang et al., 2008). The pharmacological properties of the plant roots are generally attributed to the presence of such iridoid glycosides as catalpol, aucubin and harpagide (Zhang et al., 2008), which have been shown to have a range of biological activities. They have demonstrated antitumor activity by inhibiting the

\footnotetext{
*Corresponding author, email: ewelina.piatczak@umed.lodz.pl
}

enzyme topoisomerase I, involved in tumoral processes (Galvez et al., 2005), and possessing cytotoxic activity toward lung cancer cell lines (Hung et al., 2008). Additionally, catalpol possesses extensive ischemic neural protection (Li et al., 2005), increases brain angiogenesis (Zhu et al., 2010) and exhibits hypoglycemic and diuretic activities (Zhang et al., 2008). Harpagide expresses leishmanicidal activities (Tasdemir et al., 2008) and is known to suppress lipopolysaccharide-induced nitric oxide synthase and cyclooxygenase-2 (COX-2) expression through inhibition of nuclear factor $\mathrm{kB}$ activation (Georgiev, 2013). Roots of $R$. glutinosa also accumulate phenylethanoid glycosides, including verbascoside and isoverbascoside.

Several studies describe production of metabolites characteristic for normal roots of $R$. glutinosa, using plant tissue culture such as callus cultures (Shoyama et al., 1986; Piatczak et al., 2015) and hairy root cultures obtained by Agrobacterium rhizogenes infection (Hwang, 2005; Hwang, 2009; 
Piatczak et al., 2012). Hairy root cultures are particularly well suited for production of large amounts of biomass and bioactive compounds as they offer fast growth in hormone-free media, the ability to synthesize various chemical compounds and the possibility of growth in a bioreactor. In addition, accumulation of bioactive compounds in transformed roots can be increased, e.g., by elicitation (Rao and Ravishankar, 2002).

Biosynthesis of many secondary metabolites in plants usually begins as a result of a defense response. A number of elicitors, including jasmonic acid (Zhang and Guo, 2006), its derivative methyl jasmonate (MeJa), and salicylic acid (SA) act as important plant signal transduction molecules (Chetana and Ramawat, 2009; d Onofrio et al., 2009); these are involved in stimulation of biochemical reactions which encourage the formation of low molecular weight defensive compounds in response to attack by pathogens (Ozawa et al., 2000; Schenk et al., 2000). The exogenous addition of such elicitors to plant cell culture or intact plants stimulates biosynthesis of a wide range of secondary metabolites including glucosinolates, terpenoids, phenylpropanoids, valepotriates, saponins and alkaloids (Van der Fits and Memelink, 2000; Memelink et al., 2001; de Costa et al., 2013; Russowski et al., 2013). However, elicitation is a highly complex process and depends on many factors such as elicitor concentration, growth stage of the culture, the time at which the elicitor is added and contact time with the elicitor (Namdeo, 2007). These parameters need to be optimized according to the plant species and type of bioactive compound.

The present study evaluates the influence of individually applied or combined elicitors: SA and MeJa on production of biomass, as well as iridoids (catalpol, harpagide, catalposide) and phenylethanoids (verbascoside, isoverbascoside), in hairy roots of $R$. glutinosa. The optimal concentrations and exposure times of the elicitors are also determined.

\section{MATERIALS AND METHODS}

\section{CHARACTERIZATION OF GROWTH KINETICS}

Hairy roots of $R$. glutinosa (RS-2 line) were obtained after transformation with Agrobacterium rhizogenes (strain A4) as described by Piatczak et al. (2012). The cultures were grown in Erlenmeyer flasks $(300 \mathrm{~mL})$ containing $80 \mathrm{~mL}$ of liquid WPM (Woody Plant medium) (Lloyd and McCown 1980) without any growth regulators, supplemented with $3 \%$ of sucrose, in darkness at $26^{\circ} \mathrm{C} \pm 2{ }^{\circ} \mathrm{C}$ on a rotary shaker $(70 \mathrm{rpm})$. The cultures were subcultured every 30 days. The average fresh weight
(FW) of inoculum was $0.48 \pm 0.03$ g/flask and dry weight (DW) was $0.03 \pm 0.006 \mathrm{~g} /$ flask. For kinetics studies, $R$. glutinosa hairy root cultures after 25 subcultures were cultured for 60 days, with root growth being determined every 5 days on the basis of fresh and dry weights. At each time point, the growth of hairy roots was measured in terms of FW and DW (g per flask). FW was measured after separating of hairy roots growing in the Erlenmeyer flasks from the media and washing them with distilled water. Then they were dried on some absorbent paper and weighted on laboratory scales with accuracy of $0.01 \mathrm{~g}$. For determination of DW, roots were divided into pieces of 300-600 $\mathrm{mg}$, placed on aluminium caps and weighted with $0.0001 \mathrm{~g}$ accuracy. Then they were dried for an hour at $100^{\circ} \mathrm{C}$ and for 24 hours at $80^{\circ} \mathrm{C}$ in a laboratory oven and weighted. The experiments were repeated three times (3 flasks with roots for one experiment) for three consecutive subculture cycles.

The results are presented in Fig. 1. The specific growth rate $(\mu)$ and doubling time (dt) were also estimated from the exponential phase of growth, as indicated by Wodnicka et al. (2000).

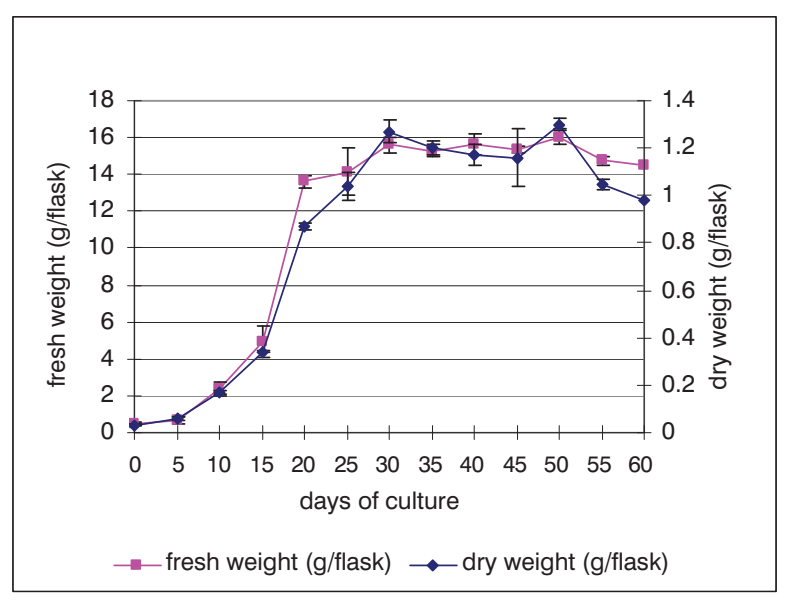

Fig. 1. Time course study of $R$. glutinosa hairy roots. Fresh and dry weight of biomass was recorded for 60 days at 5-day intervals.

Data are represented as the mean \pm standard error (SE) from three independent experiments. For each experiment 3 Erlenmeyer flasks were taken at each time point.

\section{EXPERIMENTS WITH ELICITORS}

Methyl jasmonate (MeJa) (95\% purity, Sigma Aldrich) and salicylic acid (SA) (95\% purity, Sigma Aldrich) solutions were prepared by dissolution in $96 \%$ ethanol. The solutions were sterilized by syringe filter (Merck Millipore Ltd., pore size $\varnothing 20 \mu \mathrm{m}$ ) and added to flasks containing $80 \mathrm{~mL}$ of autoclaved liquid medium with hairy root cultures to reach final concentrations of 50, 100, 150 and $200 \mu \mathrm{M}$ (the total ethanol added was $80 \mu \mathrm{L}$ per 
flask). In further experiments, MeJa and SA were also added in two combinations, i.e. $50 \mu \mathrm{M}$ MeJa + $50 \mu \mathrm{M} \mathrm{SA}$ and $100 \mu \mathrm{M} \mathrm{MeJa}+100 \mu \mathrm{M} \mathrm{SA}$.

The elicitor was added on the $23^{\text {rd }}$ day of culture without changing the medium, when the cells were in the late growth phase. The control hairy roots were treated with $80 \mu \mathrm{L}$ of $96 \%$ ethanol at the same time. The hairy roots were allowed to grow in the presence of the elicitor for further 72 or 120 hours before being harvested. Their FW and DW were then measured and their secondary metabolite contents were analyzed by UHPLC.

\section{EVALUATION OF IRIDOID \\ AND PHENYLETHANOID GLYCOSIDE CONTENTS}

The plant material was lyophilized, powdered and extracted as described by Piatczak et al. (2012). The UHPLC analyses were carried out on Agilent Technologies 1290 Infinity apparatus on a Zorbax Eclipse Plus C18 column $(100 \times 3.1 \mathrm{~mm}$ id; $1.8 \mu \mathrm{m}$ Agilent Technologies) (Piątczak et al., 2015). The compounds were identified according to their retention times and UV spectra by comparison with authentic standards (Piatczak et al., 2015). The contents of iridoid (catalpol, harpagide, catalposide) and phenylethanoid (verbascoside, isoverbascoside) glycosides in the samples were expressed as mg of the compound per gram of dry weight $\left(\mathrm{mg} \times \mathrm{g}^{-1} \mathrm{DW}\right)$. The results are presented in Figs. 3 and 4. Finally, metabolite production $\left(\mathrm{mg} \cdot \mathrm{L}^{-1}\right)$ after MeJa treat- ment of hairy root culture was also determined according to the formula: mean DW $\left(\mathrm{g} \cdot \mathrm{L}^{-1}\right) \mathrm{x}$ mean content of each metabolite (mg. $\left.\mathrm{g}^{-1} \mathrm{DW}\right)$ (Table 1 ).

\section{STATISTICAL ANALYSIS}

All the presented results are means from three independent experiments \pm standard error (SE). The results presented in Table 1 and Figs. 2-4 were analyzed by the Kruskal-Wallis test, with $\mathrm{p} \leq 0.05$ as the significance level. STATISTICA 10 (STATSoft) Software was used for all the analyses.

\section{RESULTS AND DISCUSSION}

\section{GROWTH KINETICS IN SHAKE FLASKS}

The growth curve of $R$. glutinosa hairy roots grown in WPM liquid medium was determined based on FW and DW accumulation over a period of 60 days (Fig. 1). With regard to FW accumulation, an initial 5-day lag phase was first observed, followed by an exponential phase from day 5 to day 20 , during which the maximum growth rate was obtained and the culture reached $85 \%$ of the final fresh weight. From the exponential growth phase, a maximum specific growth rate of $\mu=0.354$ day $^{-1}$ (corresponding to $\mathrm{dt}=1.96$ days) was calculated. The values were similar to those for cultures of other plant species, for example Atropa belladon-

TABLE 1. Production of iridoid and phenylethanoid glycosides in hairy root culture of R. glutinosa after MeJa elicitation for 72 and 120 hours

\begin{tabular}{|c|c|c|c|c|c|}
\hline \multirow{2}{*}{$\begin{array}{c}\text { MeJa concentration } \\
(\mu \mathrm{M})\end{array}$} & \multirow{2}{*}{$\begin{array}{l}\text { Exposure time } \\
\text { (hours) }\end{array}$} & \multicolumn{4}{|c|}{ Metabolite production $\left(\mathrm{mg} \cdot \mathrm{L}^{-1}\right) \pm \mathrm{SE}^{*}$} \\
\hline & & Catalpol & Harpagide & Verbascoside & Isoverbascoside \\
\hline 0 (control) & & $15.71^{\mathrm{ad}} \pm 3.57$ & $0.29^{\mathrm{a}} \pm 0.01$ & $100.14^{\mathrm{a}} \pm 6.67$ & $4.58^{\mathrm{a}} \pm 0.79$ \\
\hline 50 & & $3.64^{\mathrm{b}} \pm 1.45$ & $0.70^{\mathrm{b}} \pm 0.34$ & $679.04^{\mathrm{bc}} \pm 50.58$ & $8.31^{\mathrm{b}} \pm 0.90$ \\
\hline 100 & 72 & $3.09^{b} \pm 0.33$ & $1.22^{\mathrm{c}} \pm 0.01$ & $712.70^{\mathrm{b}} \pm 41.88$ & $10.25^{c} \pm 0.79$ \\
\hline 150 & & $4.89^{c} \pm 0.48$ & $1.86^{\mathrm{d}} \pm 0.01$ & $677.07^{c} \pm 28.92$ & $10.35^{c} \pm 0.23$ \\
\hline 200 & & $3.85^{\mathrm{b}} \pm 0.54$ & $1.83^{\mathrm{d}} \pm 0.07$ & $845.45^{d} \pm 5.64$ & $24.94^{\mathrm{d}} \pm 7.38$ \\
\hline 0 (control) & & $17.15^{\mathrm{a}} \pm 3.57$ & $0.30^{\mathrm{a}} \pm 0.06$ & $104.17^{\mathrm{a}} \pm 11.08$ & $7.34^{\mathrm{bg}} \pm 1.15$ \\
\hline 50 & & $14.17^{\mathrm{ad}} \pm 2.15$ & $0.63^{\mathrm{b}} \pm 0.17$ & $465.92^{\mathrm{e}} \pm 84.88$ & $18.36^{\mathrm{e}} \pm 2.51$ \\
\hline 100 & 120 & $22.51^{\mathrm{e}} \pm 0.87$ & $0.80^{\mathrm{b}} \pm 0.39$ & $348.07^{\mathrm{f}} \pm 65.64$ & $4.77^{\mathrm{a}} \pm 2.07$ \\
\hline 150 & & $29.68^{\mathrm{f}} \pm 3.57$ & $1.30^{\mathrm{c}} \pm 0.06$ & $412.95^{\mathrm{ef}} \pm 5.59$ & $12.95^{\mathrm{f}} \pm 2.18$ \\
\hline 200 & & $12.69^{\mathrm{d}} \pm 2.12$ & $0.92^{\mathrm{cb}} \pm 0.12$ & $280.40^{\mathrm{h}} \pm 32.87$ & $6.65^{g} \pm 0.74$ \\
\hline
\end{tabular}

* Production was expressed in mg of each metabolite per liter of medium. Presented data are the means from three replicates \pm standard error (SE). Presented values followed by the same letter within each column are not statistically different at $\mathrm{p} \leq 0.05$ according to the Kruskal-Wallis test. 
na (Kanokwaree and Doran, 1997) and Nicotiana tabacum (Wongsamuth and Doran, 1997). Beyond day 20 , the rate of hairy root growth was reduced. A stationary phase was identified between days 30 and 50, in which biomass remained generally unchanged. However, this began to decrease after day 50, probably due to the depletion of nutrients in the culture medium. A similar pattern was shown by DW (Fig. 1). The highest values for biomass obtained in shake flasks were $16.04 \mathrm{~g} /$ flask (FW) and $1.30 \mathrm{~g} /$ flask (DW) at day 50 of culture.

\section{EFFECT OF MEJA AND SA ON ROOT BIOMASS AND METABOLITE PRODUCTION}

Figures 2a, b and 3 show the individual effects of MeJa and SA on biomass accumulation and level of secondary metabolites (catalpol, harpagide, verbascoside, isoverbascoside) in hairy root culture of $R$. glutinosa. MeJa significantly decreased fresh and dry weight of roots compared to the controls (untreated roots), especially at higher concentrations ( 150 and $200 \mu \mathrm{M}$ ). Maximum reduction in root biomass was observed at the highest MeJa concentration $(200 \mu \mathrm{M})$ during the $120 \mathrm{~h}$ exposure to the elicitor. In the culture, fresh and dry weights of roots decreased to 12.70 and 1.02 g/flask, respectively, which were $20 \%$ lower than those in the control roots ( $16.05 \mathrm{~g} \mathrm{FW/flask}$ and $1.27 \mathrm{~g}$ DW/flask). At a shorter exposure time (72 hours) to $200 \mu \mathrm{M} \mathrm{MeJa}$, the fresh (14.49 g/flask) and dry weights $(1.13 \mathrm{~g} /$ flask) of root biomass were only $10 \%$ and $15 \%$ lower than the control roots ( $16.15 \mathrm{~g}$ FW/flask and $1.33 \mathrm{~g} \mathrm{DW/flask)} \mathrm{(Fig.} \mathrm{2a,} \mathrm{b).}$

The application of SA (50-200 $\mu \mathrm{M})$ also inhibited the growth of $R$. glutinosa hairy roots in respect to both fresh and dry biomass (Fig. 2a, b). In general, biomass accumulation in root cultures treated with SA was lower than that at the same MeJa concentrations. For example, the fresh root biomass (9.60 g/flask) after $72 \mathrm{~h}$ incubation with $200 \mu \mathrm{M}$ SA was $40.6 \%$ lower than that in the control (16.15 g/flask) and $33.8 \%$ lower compared to that of treatment with $200 \mu \mathrm{M}$ MeJa over the same time. In our study, SA (50-200 $\mu \mathrm{M})$ also caused browning and necrosis of $R$. glutinosa root tissues, whereas there was no apparent change in the color and morphology of the roots treated with MeJa. It may be due to direct toxic effect of SA and loss of viability of the culture. Such an effect of SA was also observed in Salvia miltiorrhiza cell culture (Dong et al., 2010) and Taxus baccata cell culture (Rezaei et
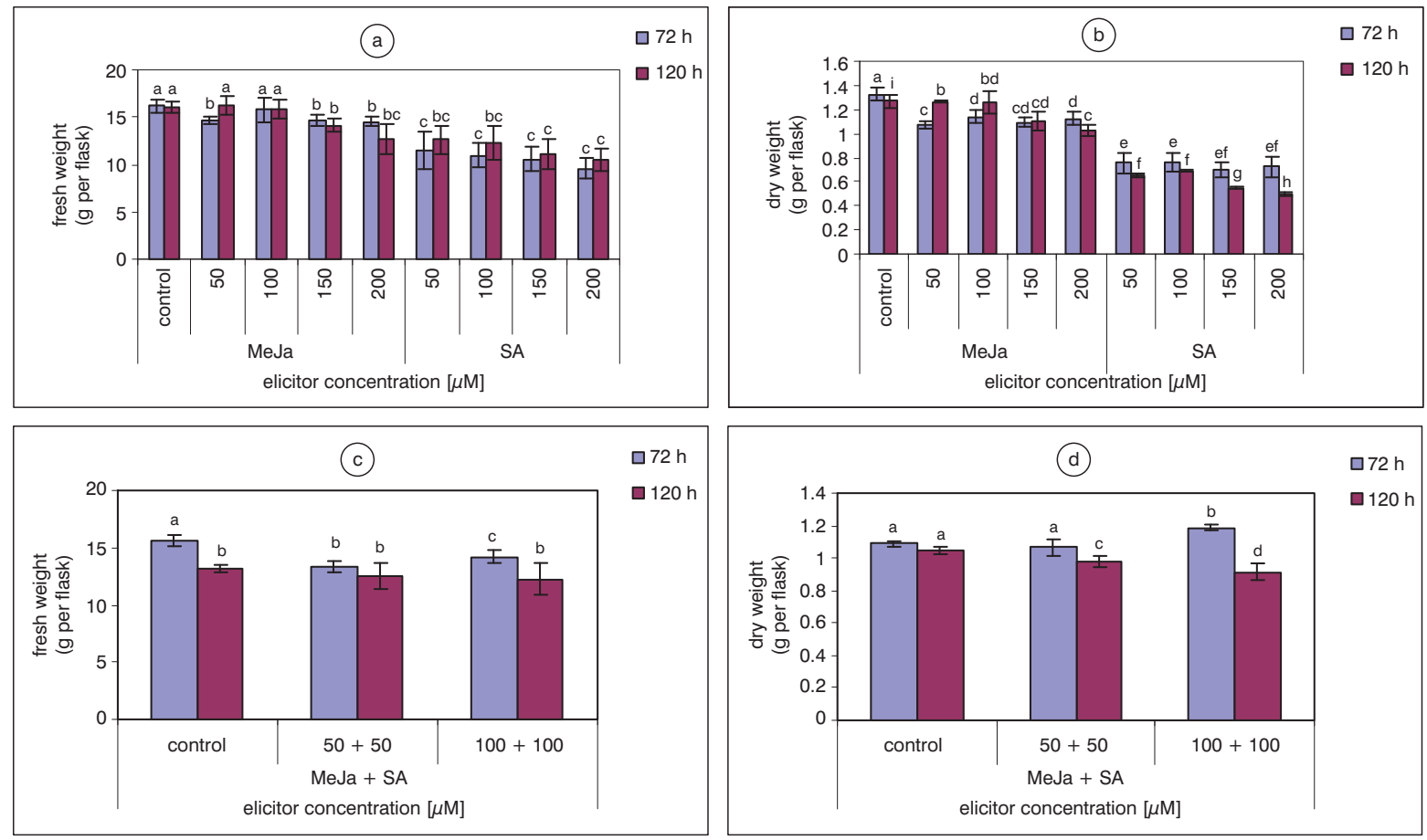

Fig. 2. Effect of different MeJa and SA concentrations alone (a, b) or combined (c, d) on accumulation of (a, c) fresh, (b, d) dry weights in g/flask in $R$. glutinosa hairy root cultures at two different times of exposure to elicitor (72 and 120 hours). The elicitor was added to 23-day-old culture (average FW was $15.3 \pm 1.2 \mathrm{~g} /$ flask and average DW was $1.3 \pm 0.002$ ). Data are represented as means from three independent experiments ( 3 flasks per single experiment) \pm standard error (SE). Presented values followed by the same letter are not statistically different at $\mathrm{p} \leq 0.05$ according to the Kruskal-Wallis test. 
al., 2011). In general, elicitation by chemical elicitors such as MeJa or SA has a negative effect on biomass accumulation leading to low overall productivity in in vitro cultures. Decrease in biomass accumulation in the presence of MeJa and SA has been earlier reported in Panax ginseng adventitious roots (Kim et al., 2004), Scopolia parviflora adventitious roots (Kang et al., 2004) and Eleuterococcus koreanum adventitious roots (Lee et al., 2015). Moreover, Lee et al. (2015) also reported that adventitious roots of $E$. koreanum treated with high MeJa concentration $(400 \mu \mathrm{M})$ showed better growth than at high SA concentration $(400 \mu \mathrm{M})$. To minimalize the negative effect of elicitors on biomass accumulation in the present study, we added the elicitor (MeJa or SA) on the $23^{\text {rd }}$ day of cultivation, when the root culture was found to be at late growth stage (Fig. 1). Earlier, a strategy of using the elicitor after the end of intensive culture growth or at the stationary phase was employed by Bonfill et al. (2011) for increased production of centelloside in Centella asiatica cell culture. Also, Cui et al. (2012) showed that addition of the elicitor at the late exponential growth phase could increase the valtrate content in Valeriana amurensis adventitious root culture without significantly affecting the biomass. According to James et al. (2008), when intensive growth had ended secondary metabolites rather than primary ones, were synthesized.

Our study showed that metabolite profile in hairy roots of $R$. glutinosa elicited with MeJa or SA remained the same as in the unelicited roots. However, the quantity of the phytochemicals was affected by the elicitation procedure. We found that the content of each metabolite tested was considerably higher compared to the control (untreated roots), when the root culture was treated with MeJa (Fig. 3). The efficiency of the elicitor varies depending on its concentration (50-200 $\mu \mathrm{M})$ and exposure time $(72 \mathrm{~h}$ or $120 \mathrm{~h})$. Both parameters need to be optimized for each individual compound. This conclusion agrees with these reached for several other metabolites and in vitro culture systems (Namdeo, 2007; Sivanandhan et al., 2013). Among concentrations tested, $150 \mu \mathrm{M}$ MeJa was the best for biosynthesis of iridoids (catalpol, harpagide) (Fig. 3a, b), whereas for maximum improvement of phenylethanoid (verbascoside and isoverbascoside) content $200 \mu \mathrm{M}$ MeJa was required (Fig. 3c, d).

The maximum level of catalpol $\left(2.15 \mathrm{mg} \cdot \mathrm{g}^{-1}\right.$ DW) was achieved in the roots treated with $150 \mu \mathrm{M}$ MeJa for $120 \mathrm{~h}$. The amount was 2 times higher compared to the control. Treatment of roots with the same concentration of MeJa $(150 \mu \mathrm{M})$ but for
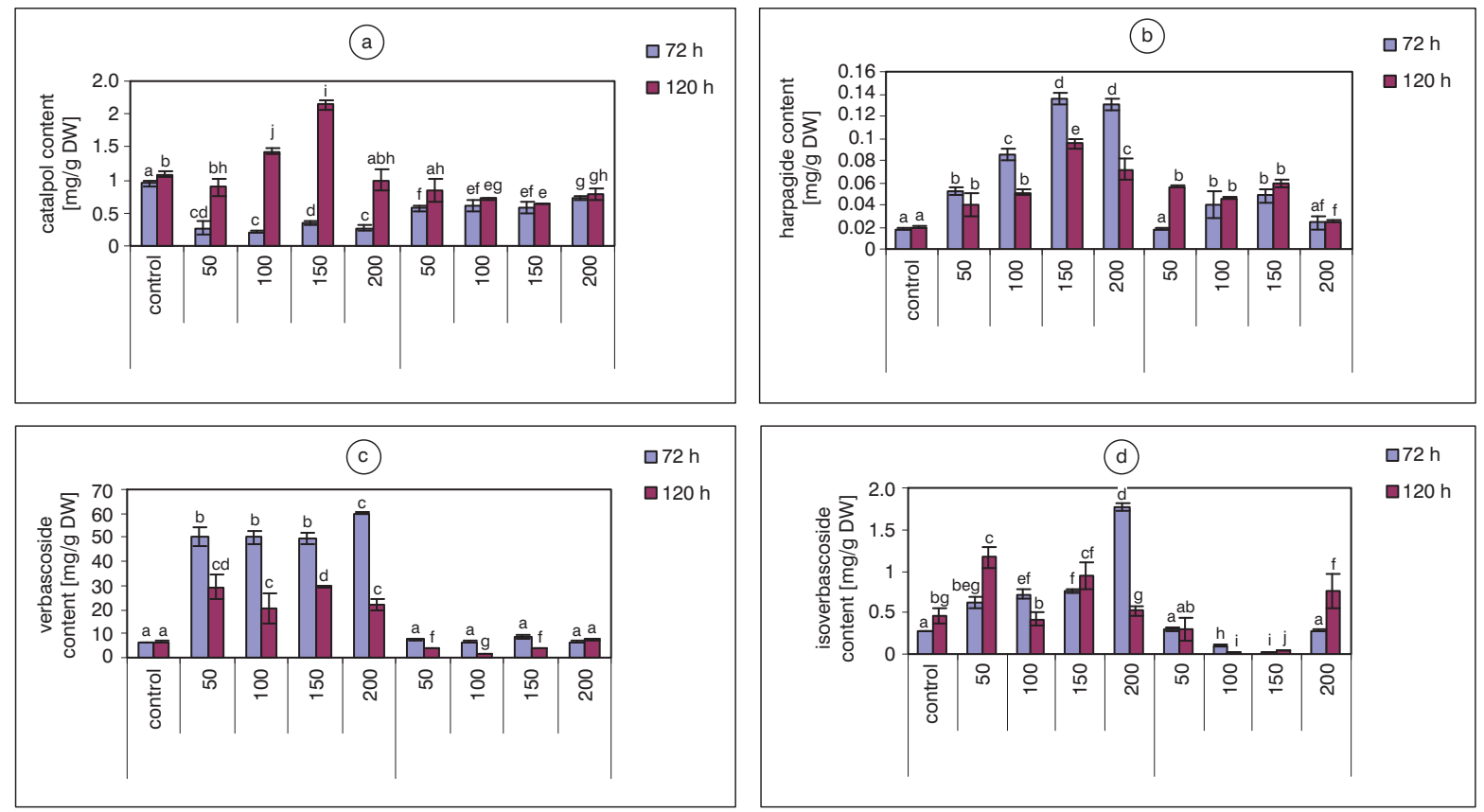

Fig. 3. Effect of different MeJa and SA concentrations on accumulation of catalpol (a), harpagide (b), verbascoside (c) and isoverbascoside (d) in $R$. glutinosa hairy root culture.

The elicitor was added to 23-day-old culture and roots were harvested after 72 and 120 hours. Data are represented as means from three replicates \pm standard error (SE). Presented values followed by the same letter are not statistically different at $\mathrm{p} \leq 0.05$ according to the Kruskal-Wallis test. 
$72 \mathrm{~h}$ reduced the catalpol content below the control (Fig. 3a). $150 \mu \mathrm{M}$ MeJa and $120 \mathrm{~h}$ exposure time were also found to be favorable for production of catalposide (6-O-p-hydroxybenzoyl catalpol), whose carbon skeleton is closely related to catalpol. Its amount increases from traces (in control roots) to $0.04,0.07$ and $0.093 \mathrm{mg} \cdot \mathrm{g}^{-1} \mathrm{DW}$ at the concentration of 50, 100 and $150 \mu \mathrm{M}$ MeJa, respectively. The greatest level of harpagide was detected in the roots treated with $150 \mu \mathrm{M}$ MeJa within short treatment duration $(72 \mathrm{~h})$. The roots produced $0.136 \mathrm{mg} \cdot \mathrm{g}^{-1} \mathrm{DW}$ of harpagide, i.e. 7.5-fold more than in the control culture without the elicitor (Fig. $3 \mathrm{~b}$ ). The amount of the iridoid declined by about $30 \%$ when the exposure time to the elicitor was prolonged to $120 \mathrm{~h}$. It might be a result of either secondary metabolite degradation in situ or its leakage into the medium. Kang et al. (2004) reported release of tropane alkaloids from roots of Scopolia parviflora into the culture medium as a result of treatment with MeJa.

Treatment with the highest concentration of MeJa $(200 \mu \mathrm{M})$ in a short period $(72 \mathrm{~h})$ was needed to maximize accumulation of phenylethanoid glycosides in hairy root culture of $R$. glutinosa. Under these conditions verbascoside and isoverbascoside levels of $60.07 \mathrm{mg} \cdot \mathrm{g} \mathrm{DW}{ }^{-1}$ and $1.77 \mathrm{mg} \cdot \mathrm{g} \mathrm{DW}^{-1}$ were obtained, which were, respectively, 10 and 6.4 times higher than those in the control.

Despite the fact that MeJa fed to the hairy root culture suppressed the biomass, increment in secondary metabolite production (calculated as $\mathrm{mg}$ per liter of medium) in the root culture treated with MeJa was observed; at optimal conditions of elicitation production of verbascoside, isoverbascoside, catalpol and harpagide in hairy roots of $R$. glutinosa increased up to 8.4-, 5.4-, 1.7- and 6.4-fold, respectively, as compared to the control (Table 1).

Our results clearly suggested that phenylethanoids, especially verbascoside were more responsive to MeJa treatment than iridoids. It is known that verbascoside production is often enhanced by both biotic and abiotic stress conditions, e.g., elicitation (Chen et al., 2007; Marsh et al., 2014). Matsumoto et al. (1987) suggested that verbascoside and its derivatives play an important role in the defense mechanism of plants and may act as a resistant component or a protector against attacks by fungi or viruses. No reports have documented catalpol-type or harpagide-type iridoid production in in vitro cultures by employing a chemical elicitor. The compounds which belong to terpenoid group use different metabolic pathways than those used by phenylethanoid glycosides, as verbascoside and isoverbascoside. Therefore, the effect of MeJa on both groups of compounds may be different. Hayashi et al. (2003) showed that stimulated effects of MeJa on production of various secondary metabolites are at least partially due to activation of key enzymes, which catalyze their biosynthesis. The time required for reaching maximal levels of the enzyme activity is different for each of biosynthetic pathways. Therefore, the amounts of metabolites varied with duration of incubation period with the elicitor.

Fig. 3 also shows the effects of different concentrations of SA on accumulation of iridoids and phenylethanoids in hairy root culture of $R$. glutinosa. It was found that SA elicitation was less effective than MeJa elicitation in producing secondary metabolites. When compared to the control culture, addition of SA led to significant decrease of catalpol content at all the concentrations tested and both used times of exposure (72 and $120 \mathrm{~h}$ ). Treatment with different concentrations of SA increased harpagide content in almost all root cultures as compared with the untreated roots but the increase was not as high as maximum level obtained by MeJa elicitation (Fig. 3b). A similar trend was observed in respect to phenylethanoid biosynthesis. At optimal conditions of SA elicitation, i.e. $150 \mu \mathrm{M}$ of the elicitor concentration and $72 \mathrm{~h}$ exposure time verbascoside level ( $8.45 \mathrm{mg} \cdot \mathrm{g}^{-1} \mathrm{DW}$ ) was only 1.4 -fold higher in comparison to the control $\left(6.04 \mathrm{mg}^{-1} \mathrm{~g}^{-1} \mathrm{DW}\right)$ and up to 5.8 times lower than that in the roots treated with the same concentration of MeJa at the same exposure time. Likewise, Gueven and Knorr (2011) reported that MeJa was more effective than SA for production of isoflavones in soybean cell suspension culture.

The inability of SA to increase the content of metabolites in hairy root culture of $R$. glutinosa to the magnitude of that caused by MeJa could be attributed to the difference in the modes of action of the two kinds of signaling transducers. While both compounds regulate pathogen-induced plant defense responses, the concentration of MeJa usually increases with insect and/or animal invasions and wounding responses. SA has been shown to be essential for systemic acquired resistance to microbial pathogens (Dong, 1998). However, it is not a universal inducer of phytoalexins (Zhao et al., 2005).

THE EFFECT OF MEJA AND SA COMBINATION ON ROOT BIOMASS AND METABOLITE CONTENT

As elicitors often display a synergistic effect on the production of secondary metabolites when applied in combination (Sahu et al., 2013), the present study also investigates the effects of combined MeJa and SA application on biomass accumulation and the content of iridoids and phenylethanoids in R. glutinosa hairy root cultures (Figs. 2c, d and 4). Two combinations of elicitors (i.e. $50 \mu \mathrm{M}$ SA + $50 \mu \mathrm{M}$ MeJa and $100 \mu \mathrm{M} \mathrm{SA}+100 \mu \mathrm{M}$ MeJa) were administered using the same procedure for MeJa 
or SA alone, i.e. elicitors were added to a 23-dayold culture and the roots were harvested after 72 and 120 hours. Both elicitor combinations slightly decreased root biomass accumulation, expect for the results of dry weight at the combination of 100 $\mu \mathrm{M} \mathrm{SA}+100 \mu \mathrm{M}$ MeJa and 72-hour period of exposure (Fig. 2c, d). In addition, combined elicitation of MeJa and SA resulted in elevated catalpol, harpagide, verbascoside and isoverbascoside content, compared to the control culture, but these values were not as high as the maximum levels obtained following elicitation by MeJa alone.

Likewise, elicitation of metabolite production by single elicitors was found to be dependent on the elicitor concentration and contact period. The highest verbascoside content (9.7 times higher than in the control) was detected in the roots elicited by 72-hour treatment with $50 \mu \mathrm{M} \mathrm{SA}+50 \mu \mathrm{M}$ MeJa (Fig. 4c). However, the greatest level of isoverbascoside and harpagide was observed after treatment with a combination of higher levels of SA and MeJa $(100 \mu \mathrm{M} \mathrm{SA}+100 \mu \mathrm{M}$ MeJa) for the same exposure time: production being almost 4-5 times higher than observed in the untreated roots. The highest quantities of catalpol (1.7 times more than in the control) were observed in the roots treated with the same combination of elicitors for 120 hours. In addition, the combination of MeJa and SA stimulated catalpol accumulation at an earlier stage of elicitation (72 hours) than MeJa alone, where increased production was observed only after a longer period of exposure ( 120 hours).

\section{CONCLUSIONS}

Our results showed that iridoid (catalpol, harpagide, catalposide) and phenylethanoid (verbascoside, isoverbascoside) glycoside content was substantially enhanced when MeJa at optimal concentration ( 150 and $200 \mu \mathrm{M}$ ) was added to the 23-day-old hairy root culture of $R$. glutinosa. Among the compounds verbascoside was accumulated at the highest amount $\left(60.07 \mathrm{mg} \cdot \mathrm{g}^{-1} \mathrm{DW}\right.$; up to 10 times more than in the control). The concentration of secondary metabolites was also increased in response to MeJa combined with SA. However, SA alone showed no substantial increase in their levels. The results suggest that MeJa and SA have different models of action on transformed root culture of $R$. glutinosa. This is the first report on the use of elicitation strategy on enhancement in bioactive metabolite production in hairy root culture of R. glutinosa.
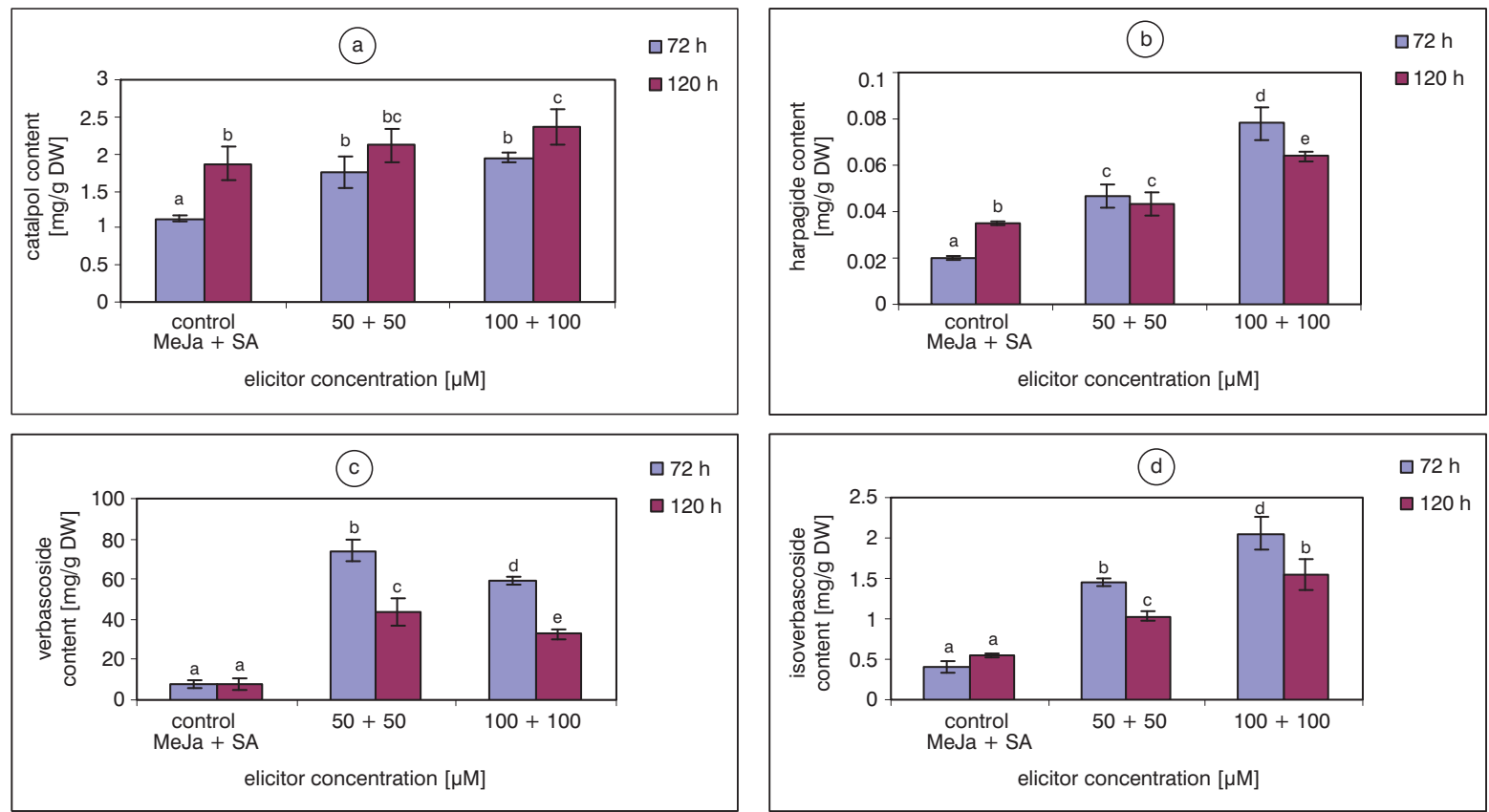

Fig. 4. Effect of combined MeJa and SA treatment on accumulation of catalpol (a), harpagid (b), verbascoside (c) and isoverbascoside (d) in $R$. glutinosa hairy roots cultures at two different times of exposure to elicitor.

The elicitors were added to 23-day-old culture and roots were harvested after 72 and 120 hours. Presented data are the means of three replicates \pm standard error (SE). Values followed by the same letter are not statistically different at $\mathrm{p} \leq 0.05$ according to the Kruskal-Wallis test. 


\section{AUTHORS' CONTRIBUTIONS}

EP designed and carried out all the elicitation experiments, prepared the plant extracts, analyzed the data and wrote the manuscript, $€ K$ was responsible for UHPLC analyses of the plant material, HW was responsible for verification of the paper. All Authors declare that they have no conflicts of interest.

\section{ACKNOWLEDGEMENTS}

This study was supported by the Department of Biology and Pharmaceutical Botany (503/3-012 01/503-31-001), Medical University of Łódź, Poland.

\section{REFERENCES}

Angiosperm Phylogeny Group. 2009. An update of the Angiosperm Phylogeny Group classification for the orders and families of flowering plants: APG III. Botanical Journal of Linnean Society 161: 105-121.

Bonfill M, Mangas S, Moyano E, Cusido RM, and Palazon J. 2011. Production of centellosides and phytosterols in cell suspension cultures of Centella asiatica. Plant Cell Tissue Organ Culture 104: 61-67.

Chen WH, Xu HM, Zeng JL, Zhao B, Wang XD, and Wang YC. 2007. Improvement of echinacoside and acteoside production by two-stage elicitation in cell suspension culture of Cistanche deserticola. World Journal of Microbiology and Biotechnology 23: 1451-1458.

ChETANA R, AND RAMAWAT KG. 2009. Elicitor induced accumulation of stilbenes in cell suspension cultures of Cayratia trifolia (L.) Domin. Plant Biotechnology Reports 3: 135-138.

Cui L, WANG ZY, and ZHou XH. 2012. Optimization of elicitors and precursors to enhanced valtrate production in adventitious roots of Valeriana amurensis Smir ex Kom. Plant Cell Tissue and Organ Culture 108: 411-420.

de Costa F, Alves Yendo AC, Fleck JD, Gosmann G, and FettNeto AG. 2013. Accumulation of a bioactive triterpene saponin fraction of Quillaja brasiliensis leaves is associated with abiotic and biotic stresses. Plant Physiology and Biochemistry 66: 56-62.

Dong X. 1998. SA, JA, ethylene and disease resistance in plants. Current Opinion in Plant Biology 1: 316-323.

Dong J, WAN G, and Liang Z. 2010. Accumulation of salicylic acid-induced phenolic compounds and raised activities of secondary metabolic and antioxidative enzymes in Salvia miltiorrhiza cell culture. Journal of Biotechnology 148: 99-104.

D'ONOFrio C, Cox A, DAvis C, and Boss PK. 2009. Induction of secondary metabolism in grape cell cultures by jasmonates. Functional Plant Biology 36: 323-338.

Galvez M, Martin-Cordero C, and Ayuso MJ. 2005. Iridoids as DNA topoisomerase I poisons. Journal of Enzyme Inhibition and Medical Chemistry 20: 389-392.

Georgiev MI, Ivanovska N, Alipieva K, Dimtrova P, and VerPOORTE R. 2013. Harpagoside: from Kalahari Desert to pharmacy shelf. Phytochemistry 92: 8-15.
Gueven A, and KnorR D. 2011. Isoflavonoid production by soy plant callus suspension culture. Journal of Food Engineering 103: 237-243.

Hayashi H, Huang P, and Inoue K. 2003. Up-regulation of soyasaponin biosynthesis by methyl jasmonate in cultured cells of Glycyrrhiza glabra. Plant and Cell Physiology 44: 404-411.

Hung JY, Yang JC, Tsai YM, and Huang MS. 2008. Antiproliferative activity of aucubin is through cell cycle arrest and apoptosis in human non-small cell lung cancer A549 cells. Clinical and Experimental Pharmacology and Physiology 35: 995-1001.

HwANG SJ. 2005. Growth characteristics and catalpol production in Chinese foxglove (Rehmannia glutinosa Libosch.) hairy roots transformed with Agrobacterium rhizogenes ATCC15834. Journal of Plant Biology 48: 380-386.

Hwang SJ. 2009. Catalpol production in Chinese foxglove (Rehmannia glutinosa Libosch.) hairy roots transformed with Agrobacterium rhizogenes ATCC15834. Methods in Molecular Biology 547: 263-273.

JAMES JT, MEYer R, and Dubery IA. 2008. Characterization of two phenotypes of Centella asiatica in Southern Africa through the composition of four triterpenoids in callus, cell suspensions and leaves. Plant Cell Tissue and Organ Culture 94: 91-99.

KANG SM, JUnG HY, KANG YM, and CHOI MS. 2004. Effects of methyl jasmonate and salicylic acid on the production of tropane alkaloids and the expression of PMT and $\mathrm{H} 6 \mathrm{H}$ in adventitious root cultures of Scopolia parviflora. Plant Science 166: 745-51.

KANOKWAREE K, and DORAN PM. 1997. The extend to which external oxygen transfer limits growth in shake flask culture of hairy roots. Biotechnology and Bioengineering 55: 520-526.

Kim YS, Hahn EJ, Hosakatee NM, and PaEk KY. 2004. Adventitious root growth and ginsenoside accumulation in Panax ginseng cultures as affected by methyl jasmonate. Biotechnology Letters 26: 1619-1622.

Kitagawa I, Fukuda Y, Taniyama T, and Yoshikawa M. 1991. Chemical studies on crude drug processing. VII. On the constituents of Rehmanniae radix. (1): Absolute stereostructures of rehmaglutins A, B and D isolated from Chinese Rehmanniae Radix, the dried root of Rehmannia glutinosa Libosch. Chemical and Pharmaceutical Bulletin 39: 1171-1176.

LEE EJ, PARK SY, and PEAK KY. 2015. Enhancement strategies of bioactive compound production in adventitious root cultures of Eleuterococcus koreanum Nakai subjected to methyl jasmonate and salicylic acid elicitation through airlift bioreactor. Plant Cell Tissue and Organ Culture 120: $1-10$.

LI DQ, Li Y, Liu Y, et al. 2005. Catalpol prevents the loss of CA1 hippocampal neurons and reduces working errors in gerbils after ischemia-reperfusion injury. Toxicon 46 : 845-851.

Marsh Z, Yang T, Nopo-Olazabal L, Wu S, Ingle T, Joshee N, and Medina-Bolivar M. 2014. Effect of light, methyl jasmonate and cyclodextrin on production of phenolic compounds in hairy root cultures of Scutellaria lateriflora. Phytochemistry 107: 50-60.

Matsumoto M, Koga S, Shoyama Y, and Nishioka I. 1987. Phenolic glycoside composition of leaves and callus cultures of Digitalis purpurea. Phytochemistry 26: 3225-3227. 
Memelink J, Verpoorte R, and KiJne W. 2001. ORCAnization of jasmonate-responsive gene expression in alkaloid metabolism. Trends in Plant Science 6: 212-219.

NAMDEO AG. 2007. Plant cell elicitation for production of secondary metabolites: a review. Pharmacognosy Reviews 1: 69-79.

OZAWA R, ARIMURA G, TAKaBayashi J, SHIMOdA T, and Nishioka T. 2000. Involvement of jasmonate- and salicylate-related signaling pathways for the production of specific herbivore-induced volatiles in plants. Plant Cell Physiology 41: 391-398.

Pharmacopoeia Commision of the People's Republic of China 2000. The pharmacopoeia of the People's Republic of China, vol. 1. Chemical Industry Publishing House, Beijing, pp. 94.

Piątczak E, Królicka A, Wielanek M, and WysokińsKa H. 2012. Hairy root cultures of Rehmannia glutinosa and the production of iridoid and phenylethanoidnglycosides. Acta Physiologiae Plantarum 34: 2215-2224.

Piatczak E, KuŹma $€$, Sitarek P, and Wysokińska H. 2015. Shoot organogenesis, molecular analysis and secondary metabolite production of micropropagated Rehmannia glutinosa Libosch. Plant Cell Tissue and Organ Culture 120: 539-549.

RAO SR, and RAVISHANKAR GA. 2002. Plant cell cultures: chemical factories of secondary metabolites. Biotechnology Advances 20: 101-153.

Rezaei A, Ghanati F, and Dehaghi MA. 2011. Stimulation of taxol production by combined salicylic acid elicitation and sonication in Taxus baccata cell culture. International Conference on Life Science and Technology. IPCBEE vol. 3 IACSIT Press, Singapore.

Russowski D., Maurmann N, Rech SB, and FetT-Neto AG. 2013. Improved production of bioactive valepotriates in whole-plant liquid cultures of Valeriana glechomifolia. Industrial Crops and Products 46: 253-257.

Sahu R, Gongopadhyaj M, and Dewanjee S. 2013. Elicitorinduced rosmarinic acid accumulation and secondary metabolism enzyme activities in Solonostemon scutellarioides. Acta Physiologiae Plantarum 35: 1473-1481.

SCHENK PM, KAZAN K, Wilson I, ANDERSON JP, Richmond T, Somerville SC, and Manners JM. 2000. Coordinated plant defense responses in Arabidopsis revealed by microarray analysis. Proceedings of the National Academy of Sciences of the USA 97: 11655-11660.
Sivanandhan G, Rajesh M, Arun M, Jeyaraj M, Dev GK, ARjunan A, Manickavasagam M, Muthuselvam M, Selvarau N, and Ganapathi A. 2013. Effect of culture conditions, cytokinins, methyl jasmonate and salicylic acid on the biomass accumulation and production of withanolides in multiple shoot culture of Withania somnifera (L.) Dunal using liquid culture. Acta Physiologiae Plantarum 35: 715-728.

Shoyama Y, Matsumoto M, and Nishioka I. 1986. Four caffeoyl glycosides from callus tissue of Rehmannia glutionosa. Phytochemistry 25: 1633-1636.

Tasdemir D, Brun R, Franzblan SG, Sezgin Y, and Calis I. 2008. Evaluation of antiprotozoal and antimycobacterial activities of the resin glycosides and the other metabolites of Scrophularia cryptophila. Phytomedicine 15: 209-215.

Wodnicka M, Guarino RD, Hemperly JJ, Timmins MR, STitT D, and PitnaR JB. 2000. Novel fluorescent technology platform for high throughput cytotoxicity and proliferation assays. Journal of Biomolecular Screening 5: 141-152.

WongSAMUTH R, and Doran PM. 1997. Production of monoclonal antibodies by tobacco hairy roots. Biotechnology and Bioengineering 54: 401-415.

VAN Der Fits L, and MEMelinK J. 2000. ORCA3, a jasmonateresponsive transcriptional regulator of plant primary and secondary metabolism. Science 289: 295-297.

Yokimune Y, Hara Y, Nomura E, Seto H, and Yoshida S. 2000 The configuration of methyl jasmonate affects paclitaxel and baccatin III production in Taxus cells. Phytochemistry 54: 13-17.

ZHANG J, and GuO Z. 2006. Effect of methyl jasmonic acid on bacatin III biosynthesis. Tsinghua Science and Technology 11: 363-367.

ZHANG RX, Li MX, and JiA ZP. 2008. Rehmannia glutinosa: review of botany, chemistry and pharmacology. Journal of Ethnopharmacology 117: 199-214.

ZHAO J, DAVIS LC, and VERPOORTE R. 2005. Elicitor signal transduction leading to production of plant secondary metabolites. Biotechnology Advances 23: 283-333.

Zhu HF, Wan D, Luo Y, Zhou JL, Chen L, and Xu XY. 2010 Catalpol increases brain angiogenesis and up-regulates VEGF and EPO in the rat after permanent middle cerebral artery occlusion. International Journal of Biological Sciences 6: 443-453. 